\title{
NEURAL NETWORK BASED FEEDBACK LINEARIZATION CONTROL OF A SERVO-HYDRAULIC VEHICLE SUSPENSION SYSTEM
}

\author{
Jimoh Olarewaju PEDRO, Olurotimi AKintunde DAHUNSI
}

\author{
School of Mechanical, Aeronautical and Industrial Engineering \\ University of the Witwatersrand, 1 Jan Smuts Ave, Private Bag 03, WITS 2050, Johannesburg, South Africa \\ e-mail: Jimoh.Pedro@wits.ac.za, Olurotimi.Dahunsi@students.wits.ac.za
}

\begin{abstract}
This paper presents the design of a neural network based feedback linearization (NNFBL) controller for a two degree-offreedom (DOF), quarter-car, servo-hydraulic vehicle suspension system. The main objective of the direct adaptive NNFBL controller is to improve the system's ride comfort and handling quality. A feedforward, multi-layer perceptron (MLP) neural network (NN) model that is well suited for control by discrete input-output linearization (NNIOL) is developed using input-output data sets obtained from mathematical model simulation. The NN model is trained using the LevenbergMarquardt optimization algorithm. The proposed controller is compared with a constant-gain PID controller (based on the Ziegler-Nichols tuning method) during suspension travel setpoint tracking in the presence of deterministic road disturbance. Simulation results demonstrate the superior performance of the proposed direct adaptive NNFBL controller over the generic PID controller in rejecting the deterministic road disturbance. This superior performance is achieved at a much lower control cost within the stipulated constraints.
\end{abstract}

Keywords: neural networks, direct adaptive control, feedback linearization control, PID control, ride comfort, suspension system, servo-hydraulics.

\section{Introduction}

The growth in active suspension system research is attributable to the progress made in vibrations research, modern control theory, instrumentation, hydraulics and pneumatics, and vehicle dynamics (Fiahlo and Balas, 2002; He and McPhee, 2005; Hada et al., 2007). A good vehicle suspension system is characterized by good ride comfort, road handling, and road holding qualities. All of these must be achieved within an acceptable range of suspension travel (Kumar and Vijayarangan, 2007; Du and Zhang, 2009).

It is difficult to simultaneously satisfy these design requirements because of their conflicting nature. Hence a trade-off between them becomes imperative. The controlled variable in this work is the suspension travel because it is one of the readily measurable signals that makes the active vehicle suspension system (AVSS) design and analysis realistic (Gao et al., 2006; Du and Zhang, 2009).

The AVSS control problem is a disturbance rejection or vibration isolation one, where the road roughness profile represents the external disturbance (Hrovat, 1997; Pedro, 2007). The AVSS is a more feasible option for suspension control because of its better system static stability and performance at low frequencies.

The main challenge in AVSS controller design is robustness against parameter and disturbance variation (Pedro, 2007; Ehtiwesh and Dorovic, 2009). Applications of optimal and robust control techniques have enabled the AVSS to achieve a better trade-off of the design parameters (Hrovat, 1997; Shen and Peng, 2003; Feng et al., 2003; Chantranuwathana and Peng, 2004).

The continued use of electro-hydraulic systems in AVSSs is due to its lower cost, high power-to-weight ratio, fast response, high stiffness and good load bearing capability. However, these benefits can only be employed in full in the presence of a robust tracking controller (Du and Zhang, 2009; Nakkarat and Kuntanapreeda, 2009; Seo et al., 2007). Moreover, the nonlinearities due to the interaction between the electro-hydraulic system and the vehicle suspension are further complicated by uncertainties related to varying operating conditions of the vehicle, e.g., irregular road excitation inputs (Feng et al., 2003; Shirahatt et al., 2008).

Several conventional and nonlinear control based methods like optimal control methods (Hassanzadeh et al., 2010; Shirahatt et al., 2008; Pedro and Mgwenya, 
2004; Pedro, 2003; Hrovat, 1997), PID (Kumar, 2008; Dahunsi et al. 2010a; 2010b), $H_{2}$ (Pedro, 2007), $H_{\infty}$ (Fallah et al., 2009; Ryu et al., 2008), $H_{2} / H_{\infty}$ (Du and Zhang, 2008; Poussot-Vassal et al., 2006), sliding mode, fuzzy logic (Salem and Aly, 2009; Yoshimura and Teramura, 2005; Sharkawy, 2005; D'Amato and Viassolo, 2000) and backstepping (Nakkarat and Kuntanapreeda, 2009; Yagiz et al., 2008) have been investigated and documented.

However, the feasibility of these controllers for practical implementation is often challenged based on the following factors: sometimes the measurement of some necessary variables or states (e.g., tire deflection) could be difficult; the control scheme could lead to non-convex optimization or other computational problems difficult to solve; and there could also arise the issue of the requirement for the controller order to be as high as that of the generalized plant (Du and Zhang, 2008; Biglarbegian et al., 2008; Christophe et al., 2005).

Gaspar et al. (2003) as well as Fialho and Balas (2002) presented a linear parameter varying (LPV) control technique for a nonlinear active vehicle suspension system with actuator dynamics. LPV theory is mainly useful to tackle measurable and bounded nonlinearities (PoussotVassal et al., 2006). LPV design is also one of the fixedgain strategies that are designed to be optimal for a nominal parameter set and specific operating conditions.

Applications of sliding mode control to AVSS control are also presented by Du and Zhang (2009), Koshkouei and Burnham (2008) as well as Al-Holou et al. (2002). Although this provides some robustness characteristics and is readily applicable to nonlinear control problems, it is commonly associated with chattering and may excite unmodelled high frequency dynamics that could affect system performance or cause instability.

Feedback linearization (FBL) is one of the methods used in nonlinear control design for regulation and tracking problem. It algebraically transforms nonlinear system dynamics into fully or partially linear ones, thereby enabling the application of linear control methods in solving the problem. FBL is normally carried out by a model based transformation and feedback, rather than by linear approximations of the dynamics (Goodwin et al., 2001; Slotine and Li, 1991; Norgaard et al., 2003; Garces et al., 2003; Seo et al., 2007).

The requirement of full-state measurements and lack of guarantee for robustness are among the drawbacks of FBL which motivate for its augmentation with appropriate intelligent control techniques: neural network (Yesildirek and Lewis, 1995; He et al., 1998; Boutalis, 2004; Kar and Behera, 2009; Poursamad, 2009), and fuzzy logic (Boukezzoula et al., 2007)).

Sliding mode based feedback linearization control was applied to active vehicle suspension by Shi et al. (2010). Vertical displacement of the vehicle body was the controlled output signal and the system modelling included actuator dynamics. The relative degree and system dimension are equal so that exact feedback linearization could be applied.

Proportional, integral and derivative (PID) based feedback linearization control was applied to an electrohydraulic servo-system by Seo et al. (2007). Good handling of nonlinearities and the fact that the application of several other nonlinear control techniques often lead to the design of higher order controllers are factors that make application of FBL attractive (Du and Zhang, 2008; Chantranuwathana and Peng, 2004; Feng et al., 2003; Shen and Peng, 2003; Goodwin et al., 2001).

The direct adaptive NNFBL controller proposed in the present work solves the control problem by approximating the nonlinear model with two separate multilayer feedforward neural networks (NNIOL), and then proceeds to solve the approximated versions of the models (Yesildirek and Lewis, 1995; He et al., 1998; Norgaard et al., 2003). It is trained off-line, thereby making it less computationally challenging in comparison with model predictive and model reference controllers.

In the work of Buckner et al. (2001), feedback linearization within an indirect adaptive intelligent control framework was applied to AVSS using the radial basis function neural network (RBFNN). Gains were updated as a result of on-line estimation and updating of the model parameters at each sample time.

Hagan and Demuth (1999) as well as Cao et al. (2008) highlighted various adaptive control properties of intelligent control techniques like neural networks (NNs), fuzzy logic, genetic algorithms and sliding mode control. NNs have found wide applications in the field of control systems because of their ability to approximate arbitrary nonlinear mappings and their highly parallel structure which allows parallel implementation, thus making it more fault tolerant than the conventional schemes. NNs also have the ability to learn and adapt on-line, and have good application in multivariable systems (Cao et al., 2008; Al-Holou et al., 2002; Jin and Yu, 2008; Eski and Yildrim, 2009).

Deng et al. (2009) utilized the constraint handling advantage of model predictive control (MPC) during the control of a feedback linearized system. A dynamic neural network was employed in modelling the plant for the input-output FBL. The controller holds good prospects because it can be implemented in real time.

In the work of Dahunsi et al. (2009), approximate predictive control (APC) was applied to a nonlinear AVSS. NN modelling was based on a multi-layer perceptron NN model and trained off-line using the LevenbergMarquardt algorithm. The NNFBL controller designs documented by Poursamad (2009) as well as Kar and Behera (2009) are based on the RBFNN architecture.

In this work, PID control will be used as a bench- 
mark because of its simplicity and wide acceptability, even though its tuning is often done intuitively. PID control becomes unsuitable for AVSS application because of its limitations in terms of robustness and non-linearity, and high loop gains (Gao, 2002). These disadvantages motivate the augmentation of PID controllers with genetic algorithms (GAs) and fuzzy logic. For example, a GA was used to obtain optimum PID gains by Feng et al. (2003) as well as Kuo and Li (1999), while Lufty et al. (2009) designed a GA-trained PID-like adaptive neuro-fuzzy inference system to control nonlinear systems.

The novelty of this paper lies in the application of the designed NNFBL controller to improve real-time system performance as well as the achievement of reduced control input throughout the process. The rest of the paper is organized as follows. Section 2 presents the system overview and the modelling of the servo-hydraulic vehicle suspension system. The design of the PID and NNFBL controllers is described in Section 3. The simulation results and discussion are presented in Section 4. Section 5 contains concluding remarks.

\section{System overview and modelling}

2.1. Physical modelling. Figure 1 shows the quartercar model of the AVSS, where $m_{s}$ is the sprung mass, $m_{u}$ is the unsprung mass (wheel assembly), $k_{s}$ is the suspension spring constant, $b_{s}$ is the suspension damping coefficient and $k_{t}$ is the wheel spring constant. The vertical displacement of the car body, wheel and the road disturbance are represented by $x_{1}, x_{2}$ and $w$, respectively. The hydraulic actuator force, $F$, is applied in between the sprung and unsprung masses.

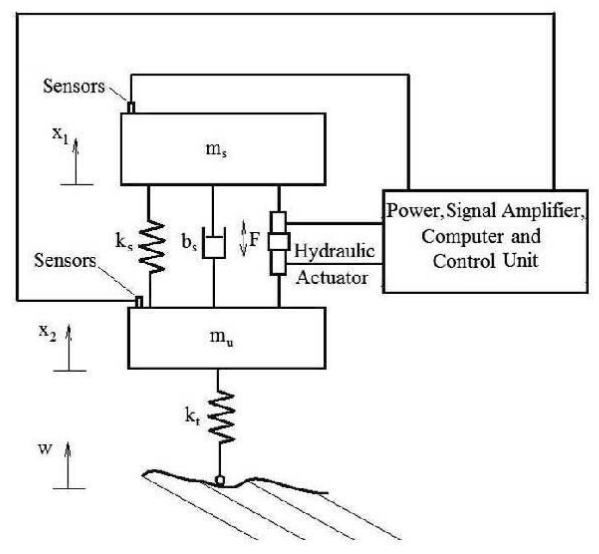

Fig. 1. Simplified quarter car model.

The relative displacement between the vehicle body and the wheel $\left(x_{2}-x_{1}\right)$ represents the suspension travel, while the relative displacement between the wheel and the road $\left(x_{2}-w\right)$ characterizes the road holding.
2.2. Mathematical modelling. Applying Newton's law to the quarter-car model shown in Fig. 1 yields the following nonlinear governing equations (Fiahlo and Balas, 2002; Gaspar et al., 2003):

$$
\begin{aligned}
m_{s} \dot{x}_{3}= & k_{s}^{l}\left(x_{2}-x_{1}\right)+k_{s}^{n l}\left(x_{2}-x_{1}\right)^{3} \\
& +b_{s}^{l}\left(x_{4}-x_{3}\right)-b_{s}^{s y m}\left|x_{4}-x_{3}\right| \\
& +b_{s}^{n l} \sqrt{\left|x_{4}-x_{3}\right|} \operatorname{sgn}\left(x_{4}-x_{3}\right)-A x_{5}, \\
m_{u} \dot{x}_{4}= & -k_{s}^{l}\left(x_{2}-x_{1}\right)-k_{s}^{n l}\left(x_{2}-x_{1}\right)^{3} \\
& -b_{s}^{l}\left(x_{4}-x_{3}\right)+b_{s}^{s y m}\left|x_{4}-x_{3}\right| \\
& -b_{s}^{n l} \sqrt{\left|x_{4}-x_{3}\right|} \operatorname{sgn}\left(x_{4}-x_{3}\right) \\
& -k_{t}\left(x_{2}-w\right)+A x_{5}, \\
\dot{x}_{5}= & \gamma \Phi x_{6}-\beta x_{5}-\alpha A\left(x_{3}-x_{4}\right), \\
\dot{x}_{6}= & \frac{1}{\tau}\left(-x_{6}+u\right),
\end{aligned}
$$

where

$$
\begin{gathered}
\alpha=\frac{4 \beta_{e}}{V_{t}}, \quad \beta=\alpha C_{t p}, \quad \gamma=C_{d} S \sqrt{\frac{1}{\rho}}, \\
\Phi=\phi_{1}+\phi_{2}, \quad \phi_{1}=\operatorname{sgn}\left[P_{s}-\operatorname{sgn}\left(x_{6}\right) x_{5}\right],
\end{gathered}
$$

and

$$
\phi_{2}=\sqrt{\left|P_{s}-\operatorname{sgn}\left(x_{6}\right) x_{5}\right|} .
$$

$A$ is the area of the piston, $x_{3}$ and $x_{4}$ are vertical velocities of the sprung and unsprung masses, respectively, $x_{5}$ is the pressure drop across the piston, $x_{6}$ is the servo valve displacement, $P_{s}$ is the supply pressure into the hydraulic cylinder, $P_{r}$ is return pressure from the hydraulic cylinder, $P_{u}$ and $P_{l}$ represent the oil pressure in the upper and lower portion of the cylinder, $V_{t}$ is the total actuator volume, $\beta_{e}$ is the effective bulk modulus of the system, $\Phi$ is the hydraulic load flow, $C_{t p}$ is the total leakage coefficient of the piston, $C_{d}$ is the discharge coefficient, $S$ is the spool valve area gradient and $\rho$ is the hydraulic fluid density.

The suspension spring and damping forces have linear and nonlinear components. Spring constant $k_{s}^{l}$ damping coefficient $b_{s}^{l}$ affect the spring force and damping force in a linear manner. Moreover, $b_{s}^{s y m}$ contributes asymmetric characteristics to the overall behaviour of the damper. Finally, $k_{s}^{n l}$ and $b_{s}^{n l}$ are responsible for the nonlinear components of the spring and damper forces, respectively.

Figure 2 illustrates the hydraulic actuator mounted in between the sprung and unsprung masses. $Q_{u}$ and $Q_{l}$ are the hydraulic fluid flow rates for the upper and the lower chambers of the hydraulic cylinder, respectively.

The actuator is controlled by means of electrohydraulic servo-valves in a three land four-way spool valve system. The maximum control input (voltage) of $10 \mathrm{~V}$ was applied to the servo-valves to achieve a maximum suspension travel of $10 \mathrm{~cm}$. 


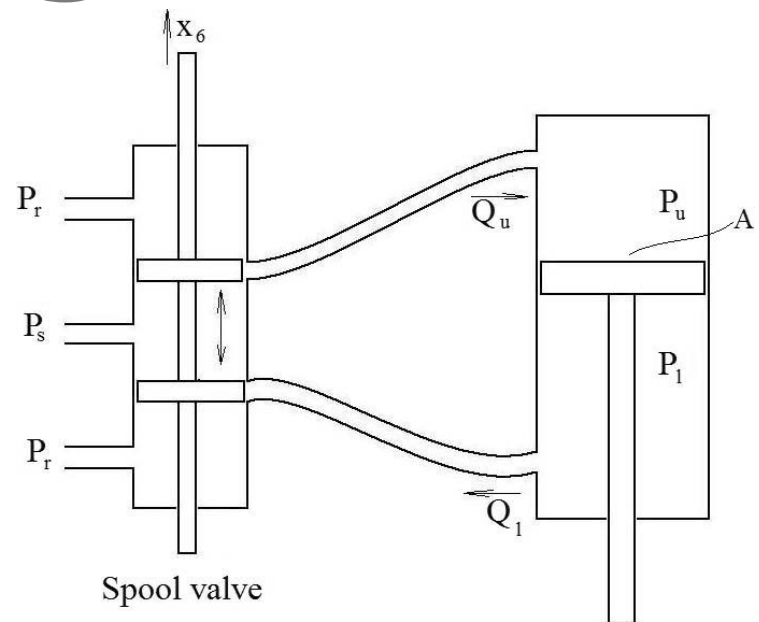

Hydraulic cylinder

Fig. 2. Schematic of the double acting hydraulic strut.

The values for the system parameters are provided in Table 1. It is assumed that the vehicle experiences a

Table 1. Parameters of the quarter-car model (Fiahlo and Balas, 2002; Gaspar et al., 2003).

\begin{tabular}{|l|c|}
\hline Parameters & Value \\
\hline \hline Sprung mass $\left(m_{s}\right)$ & $290 \mathrm{~kg}$ \\
Unsprung mass $\left(m_{u}\right)$ & $40 \mathrm{~kg}$ \\
Suspension stiffness $\left(k_{s}^{l}\right)$ & $2.35 \cdot 10^{4} \mathrm{~N} / \mathrm{m}$, \\
Suspension stiffness $\left(k_{s}^{n l}\right)$ & $2.35 \cdot 10^{6} \mathrm{~N} / \mathrm{m}$ \\
Tyre stiffness $\left(k_{t}\right)$ & $1.9 \cdot 10^{5} \mathrm{~N} / \mathrm{m}$ \\
Suspension damping $\left(b_{s}^{l}\right)$ & $700 \mathrm{Ns} / \mathrm{m}$ \\
Suspension damping $\left(b_{s}^{n l}\right)$ & $400 N \mathrm{~s} / \mathrm{m}$ \\
Suspension damping $\left(b_{s}^{\text {sym }}\right)$ & $400 \mathrm{Ns} / \mathrm{m}$ \\
Actuator parameter $(\alpha)$ & $4.515 \cdot 10^{13}$ \\
Actuator parameter $(\beta)$ & 1 \\
Actuator parameter $(\gamma)$ & $1.545 \cdot 10^{9}$ \\
Piston area $(A)$ & $3.35 \cdot 10^{-4} \mathrm{~m}{ }^{2}$ \\
Supply pressure $\left(P_{s}\right)$ & $10,342,500 \mathrm{~Pa}$ \\
Actuator time constant $(\tau)$ & $3.33 \cdot 10^{-2} \mathrm{~s}$ \\
Bump height $(a)$ & $0.11 \mathrm{~m}$ \\
Vehicle speed $(V)$ & $30 \mathrm{~ms}^{-1}$ \\
Disturbance half wavelength $(\lambda)$ & $7.5 \mathrm{~m}$ \\
\hline
\end{tabular}

sudden disturbance, a bump, whose profile is shown in Fig. 3 and is described as

$w(t)=\left\{\begin{aligned} \frac{a}{2}\left(\left(1-\cos \left(\frac{2 \pi V t}{\lambda}\right)\right),\right. & \text { if } 1.25 \leq t \leq 1.5 \\ 0, & \text { otherwise, }\end{aligned}\right.$

where $a$ is the bump height, $V$ is the vehicle speed and $\lambda$ is the half wavelength of the sinusoidal road undulation. The system of equations (1)-(4) can thus be expressed in

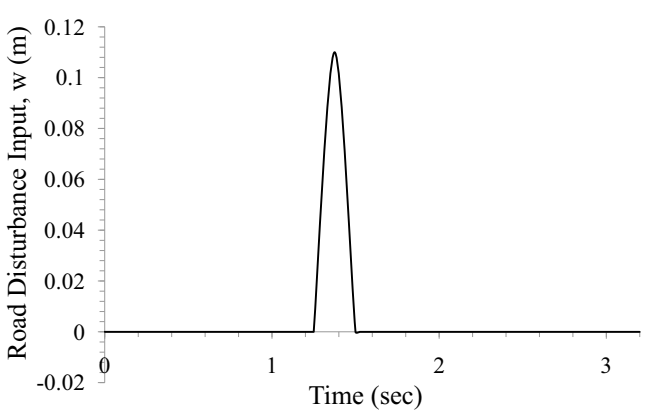

Fig. 3. Road profile.

state-space form:

$$
\begin{aligned}
\dot{\mathbf{x}} & =\mathbf{f}(\mathbf{x})+\mathbf{g}(\mathbf{x}) \mathbf{u}+\mathbf{p}(\mathbf{w}), \\
y & =h(\mathbf{x})=x_{2}-x_{1},
\end{aligned}
$$

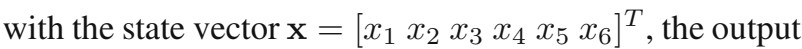
variable $y=x_{2}-x_{1}$, and the control input $\mathbf{u}$. The system matrices $\mathbf{f}$ and $\mathbf{g}$ are:

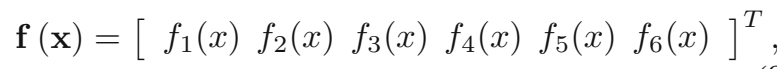

$$
\begin{aligned}
& \mathbf{g}(\mathbf{x})=\left[\begin{array}{llllll}
0 & 0 & 0 & 0 & 0 & \frac{1}{\tau}
\end{array}\right]^{T} \\
& \mathbf{p}(\mathbf{w})=\left[\begin{array}{llllll}
0 & 0 & 0 & \frac{k_{t}}{m_{u}} w & 0 & 0
\end{array}\right]^{T}, \\
& f_{1}(\mathbf{x})=x_{3}, \\
& f_{2}(\mathbf{x})=x_{4}, \\
& f_{3}(\mathbf{x})=\frac{1}{m_{s}}\left[k_{s}^{l}\left(x_{2}-x_{1}\right)+k_{s}^{n l}\left(x_{2}-x_{1}\right)^{3}\right. \\
& +b_{s}^{l}\left(x_{4}-x_{3}\right)-b_{s}^{\mathrm{sym}}\left|x_{4}-x_{3}\right| \\
& +b_{s}^{n l} \sqrt{\left|x_{4}-x_{3}\right|} \operatorname{sgn}\left(x_{4}-x_{3}\right)-A x_{5} \\
& \left.-A x_{5}\right] \text {, } \\
& f_{4}(\mathbf{x})=\frac{1}{m_{u}}\left[-k_{s}^{l}\left(x_{2}-x_{1}\right)-k_{s}^{n l}\left(x_{2}-x_{1}\right)^{3}\right. \\
& -b_{s}^{l}\left(x_{4}-x_{3}\right)+b_{s}^{\mathrm{sym}}\left|x_{4}-x_{3}\right| \\
& -b_{s}^{n l} \sqrt{\left|x_{4}-x_{3}\right|} \operatorname{sgn}\left(x_{4}-x_{3}\right) \\
& \left.-k_{t} x_{2}+A x_{5}\right] \text {, } \\
& f_{5}(\mathbf{x})=\gamma \Phi x_{6}-\beta x_{5}-\alpha A\left(x_{3}-x_{4}\right), \\
& f_{6}(\mathbf{x})=\frac{-x_{6}}{\tau} \text {. }
\end{aligned}
$$

\section{Controller implementation}

The main goal of the controller is to track a generated desired suspension travel in the presence of the deterministic road disturbance (Eqn. (5)). The controller should satisfy the following requirements: 
1. nominal stability,

2. good command tracking,

3. disturbance rejection,

4. rise time not greater than $0.1 \mathrm{~s}$, and

5. maximum overshoot not greater than $5 \%$.

6. performance index: The controller should minimize the performance index given by

$$
J=\frac{1}{t_{f}} \int_{0}^{t_{f}}\left[\left(\frac{y(t)-y_{\mathrm{ref}}(t)}{y_{\max }}\right)^{2}+\left(\frac{u(t)}{u_{\max }}\right)^{2}\right] \mathrm{d} t,
$$

where $t_{f}$ is the final time (which in this case is $5 \mathrm{~s}$ ), $y_{\text {ref }}$ is the desired suspension travel, $y_{\max }$ is the maximum allowable value of the suspension travel (controlled output), and $u_{\max }$ is the maximum allowable value of the supply voltage (control input).

3.1. PID control and tuning. The structure of the PID controller is given as (Norgaard et al., 2003; O'Dwyer, 2006)

$$
U(s)=\left(K_{p} \frac{1+T_{i} s}{T_{i} s} \frac{1+T_{d} s}{1+\alpha T_{d} s}\right) E(s),
$$

where $E(s)=Y_{\text {ref }}(s)-Y(s)$ is the error signal between the reference signal $Y_{\text {ref }}(s)$ and the actual output signal $Y(s), U(s)$ is the plant input signal, $K_{p}$ is the proportional gain, $T_{d}$ is the derivative time constant, $T_{i}$ is the integral time constant and $\alpha$ is the lag factor in the derivative component of the PID controller.

The Ziegler-Nichols tuning rule is used with a decay ratio of 0.25 to obtain the PID controller gains. PID controllers are known to often generate high control inputs which can lead to saturation. Thus, efforts were made during tuning to ensure that the control input was within the stipulated range. The PID parameters used for the simulations are given in Table 2 .

Table 2. PID tuning parameters used.

\begin{tabular}{|c|c|}
\hline Parameters & Value \\
\hline$K_{p}$ & 3.0 \\
$T_{i}$ & 0.0133 \\
$T_{d}$ & $3.8404 \cdot 10^{-3}$ \\
$\alpha$ & 0.047 \\
\hline
\end{tabular}

3.2. AVSS input-output feedback linearization. The model of the AVSS given by (6) and (7) belongs to the class of affine controls. Given that $\mathbf{x} \in \mathbb{R}^{n}$ is the state vector, $\mathbf{u} \in \mathbb{R}$ is the input vector, $\mathbf{y} \in \mathbb{R}^{m}$ is the output vector and $\mathbf{h}: \mathbb{R}^{n} \rightarrow \mathbb{R}^{m}$, f and $\mathbf{g}: \mathbb{R}^{n} \rightarrow \mathbb{R}^{n}$ are smooth functions on the state space $\mathbb{R}^{n}$. The goal of the input-output linearization is to develop a control input of the form

$$
\mathbf{u}=\mathbf{a}(\mathbf{x})+\mathbf{b}(\mathbf{x}) \nu
$$

such that there is a linear input-output mapping between the new input $\nu$ (also known as the virtual control input) and the output $\mathbf{y}$.

The first derivative of the output is

$$
\begin{aligned}
\dot{\mathbf{y}} & =\frac{\partial \mathbf{h}(\mathbf{x})}{\partial \mathbf{x}} \\
& =\frac{\partial \mathbf{h}}{\partial \mathbf{x}}[\mathbf{f}(\mathbf{x})+\mathbf{g}(\mathbf{x}) \mathbf{u}] \\
& =\mathcal{L}_{f} \mathbf{h}(\mathbf{x})+\mathcal{L}_{g} \mathbf{h}(\mathbf{x}) \mathbf{u}=x_{4}-x_{3},
\end{aligned}
$$

where $\mathcal{L}_{f} \mathbf{h}(\mathbf{x})=\frac{\partial \mathbf{h}}{\partial \mathbf{x}} \mathbf{f}(\mathbf{x})$, known as the Lie derivative of $\mathbf{h}$ along $\mathbf{f}$. Since $\mathcal{L}_{g} \mathbf{h}(\mathbf{x})=\frac{\partial \mathbf{h}}{\partial \mathbf{x}} \mathbf{g}(\mathbf{x})=0$, we have

$$
\dot{\mathbf{y}}=\mathcal{L}_{f} \mathbf{h}(\mathbf{x}) \text {. }
$$

Similarly,

$$
\begin{aligned}
\ddot{\mathbf{y}} & =\frac{\partial\left(\mathcal{L}_{f} \mathbf{h}\right)}{\partial x}[\mathbf{f}(\mathbf{x})+\mathbf{g}(\mathbf{x}) \mathbf{u}] \\
& =\mathcal{L}_{f}^{2} \mathbf{h}(\mathbf{x})+\mathcal{L}_{g} \mathcal{L}_{f} \mathbf{h}(\mathbf{x}) \mathbf{u} \\
& =\mathcal{L}_{f}^{2} \mathbf{h}(\mathbf{x})=\dot{x}_{4}-\dot{x}_{3},
\end{aligned}
$$

and $\ddot{\mathbf{y}}$ is independent of $\mathbf{u}$ since $\mathcal{L}_{g} \mathcal{L}_{f} \mathbf{h}(\mathbf{x}) \mathbf{u}=0$. Also,

$$
\begin{aligned}
\mathbf{y}^{(3)} & =\frac{\partial\left(\mathcal{L}_{f}^{2} \mathbf{h}\right)}{\partial x}[\mathbf{f}(\mathbf{x})+\mathbf{g}(\mathbf{x}) \mathbf{u}] \\
& =\mathcal{L}_{f}^{3} \mathbf{h}(\mathbf{x})+\mathcal{L}_{g} \mathcal{L}_{f}^{2} \mathbf{h}(\mathbf{x}) \mathbf{u} \\
& =\mathcal{L}_{f}^{3} \mathbf{h}(\mathbf{x})=\ddot{x}_{4}-\ddot{x}_{3},
\end{aligned}
$$

and $\mathbf{y}^{(3)}$ is also independent of $\mathbf{u}$ since $\mathcal{L}_{g} \mathcal{L}_{f}^{2} \mathbf{h}(\mathbf{x}) \mathbf{u}=0$, but

$$
\begin{aligned}
\mathbf{y}^{(4)} & =\frac{\partial\left(\mathcal{L}_{f}^{3} \mathbf{h}\right)}{\partial x}[\mathbf{f}(\mathbf{x})+\mathbf{g}(\mathbf{x}) \mathbf{u}] \\
& =\mathcal{L}_{f}^{4} \mathbf{h}(\mathbf{x})+\mathcal{L}_{g} \mathcal{L}_{f}^{3} \mathbf{h}(\mathbf{x}) \mathbf{u}
\end{aligned}
$$

and $\mathbf{y}^{(4)}$ is dependent on $\mathbf{u}$ since $\mathcal{L}_{g} \mathcal{L}_{f}^{3} \mathbf{h}(\mathbf{x}) \mathbf{u} \neq 0$. Therefore, the system's relative degree is 4 , which is less than the system dimension of 6 . Thus, it is input-output linearizable via a state feedback given by (19) and the differential homeomorphic coordinate transformation:

$$
\mathbf{z}=\mathbf{\Psi}(\mathbf{x})=\left[\begin{array}{ll}
\xi & \eta
\end{array}\right]^{T},
$$

where we have $z_{1}=y, z_{2}=\dot{y}, z_{3}=\ddot{y}, z_{4}=y^{(3)}$, $z_{5}=\psi_{1}(\mathbf{x}), z_{6}=\psi_{2}(\mathbf{x})$ so that we define $\xi=$ $\left[\begin{array}{llll}z_{1} & z_{2} & z_{3} & z_{4}\end{array}\right]^{T}, \eta=\left[\begin{array}{ll}\psi_{1}(\mathbf{x}) & \psi_{2}(\mathbf{x})\end{array}\right]^{T}$. The linearized 
system in state space form is therefore given by

$$
\begin{aligned}
\dot{\eta} & =\mathbf{f}_{\mathbf{0}}(\eta, \quad \xi), \\
\dot{\xi} & =\mathbf{A}_{\mathbf{c}} \xi+\mathbf{B}_{\mathbf{c}} \nu+\tilde{\mathbf{p}}(\mathbf{w}) \\
& =\mathbf{A}_{\mathbf{c}} \xi+\mathbf{B}_{\mathbf{c}}\left[\frac{\mathbf{u}(t)-\mathbf{a}(\mathbf{x})}{\mathbf{b}(\mathbf{x})}\right]+\tilde{\mathbf{p}}(\mathbf{w}), \\
y & =\mathbf{C}_{\mathbf{c}} \xi \\
\mathbf{A}_{\mathbf{c}} & =\left[\begin{array}{llll}
0 & 1 & 0 & 0 \\
0 & 0 & 1 & 0 \\
0 & 0 & 0 & 1 \\
0 & 0 & 0 & 0
\end{array}\right], \\
\mathbf{B}_{\mathbf{c}} & =\left[\begin{array}{llll}
0 & 0 & 0 & 1
\end{array}\right]^{T} \\
\mathbf{C}_{\mathbf{c}} & =\left[\begin{array}{llll}
1 & 0 & 0 & 0
\end{array}\right]^{T} \\
\tilde{\mathbf{p}}(\mathbf{w}) & =\left[\begin{array}{llll}
0 & 0 & 0 & 1
\end{array}\right]^{T},
\end{aligned}
$$

$\psi(\mathbf{x})$ is carefully chosen to satisfy the diffeomorphism conditions, and this requires that $\Psi$ be invertible and its derivatives be continuously differentiable. Thus (Shi et al., 2010; Isidori, 1989)

$$
\mathcal{L}_{g} \psi_{i}=\frac{\mathrm{d} \psi_{i}}{\mathrm{~d} \mathbf{x}} \mathbf{g}(\mathbf{x})=0, \quad r+1 \leq i \leq n .
$$

The unobservable states based on the controller design are the ones termed $\eta$, the zero dynamics of the system are given by the equation $\dot{\eta}=\mathbf{f}_{\mathbf{0}}(\eta, \quad \mathbf{0})$ when the origin $(\eta=\mathbf{0}, \quad \xi=\mathbf{0})$ constitutes an equilibrium point, thus making the system asymptotically stable. Equation 23) reduces to

$$
\mathbf{y}^{(4)}=\nu,
$$

implying that

$$
\mathbf{u}=\frac{1}{\mathcal{L}_{g} \mathcal{L}_{f}^{3}}\left[-\mathcal{L}_{f}^{4} \mathbf{h}(\mathbf{x})+\nu\right]
$$

(34) can be rearranged into the feedback form given in (19) such that the nonlinearities of the plant are cancelled resulting in a linear input-output relationship that is based on (33) with a transfer function:

$$
G(s)=\frac{Y(s)}{V(s)}=\frac{1}{s^{4}} .
$$

The described process of feedback linearization is illustrated in Fig. 4.

Applying a linear control design method based on the pole placement technique gives (Jelali and Kroll, 2003; Norgaard et al., 2003)

$$
\nu=-a_{r-1} y^{(r-1)}-\cdots-a_{1} \dot{y}-a_{0} y+\tilde{\nu},
$$

where $\tilde{\nu}$ is a new external input (any function of the reference signal $y_{\text {ref }}$ and the output signal $y$ ). We choose the coefficients $a_{i}$ (design parameters) such that the closedloop characteristic polynomial

$$
p(s)=s^{r}+a_{r-1} s^{r-1}+\cdots+a_{1} s+a_{0}
$$

has all its roots strictly in the open left-half complex plane. Thus the actual control input can then be written, following (34), as

$$
\mathbf{u}(t)=\frac{\tilde{\nu}(t)-\mathcal{L}_{f}^{4} \mathbf{h}(\mathbf{x})-\sum_{i=1}^{4} a_{i-1} \mathcal{L}_{f}^{i-1} \mathbf{h}(\mathbf{x})}{\mathcal{L}_{g} \mathcal{L}_{f}^{3} \mathbf{h}(\mathbf{x})} .
$$

In the present study, the desired two pairs of complex poles are selected as $s_{1,2}=-0.7 \pm 2.43 \cdot 10^{-7} j$ and $s_{3,4}=-0.75 \pm 2.67 \cdot 10^{-7} j$, based on a closed-loop characteristic polynomial given by

$$
A_{m}(s)=s^{4}+2.9 s^{3}+3.153 s^{2}+1.523 s+0.276 .
$$

If the desired output is $y_{\mathrm{ref}}(t)$, then, by introducing the tracking error,

$$
e(t)=y(t)-y_{\text {ref }}(t),
$$

and selecting the new input $\nu(t)$ such that

$$
\nu(t)=y_{\mathrm{ref}}^{(4)}(t)-C_{1} e-C_{2} \dot{e}-C_{3} \ddot{e}-C_{4} e^{(3)},
$$

where $C_{1}, C_{2}, C_{3}$ and $C_{4}$ are constants, the tracking error of the closed loop system is given by (Slotine and Li, 1991)

$$
e^{(4)}+C_{1} e+C_{2} \dot{e}+C_{3} \ddot{e}+C_{4} e^{(3)}=0,
$$

which represents exponentially stable error dynamics if perfect tracking is not achievable. The proper choice of the poles ensures that all the states remain bounded (or ensures stability in the bounded-input bounded-output (BIBO) sense) and the asymptotic output tracking error converges to zero exponentially. The system is also guaranteed for internal stability as a consequence of the global exponential stability of the zero dynamics of the system under the control law 34 when the external input $\nu(t)$ equals zero (Garces et al., 2003).

3.3. AVSS neural network based input-output feedback linearization. Direct adaptive NNFBL controller implementation consists of two steps: affine system nonlinear functions approximation $(\mathbf{f} \approx \tilde{\mathbf{f}})$ and $(\mathbf{g} \approx \tilde{\mathbf{g}})$, and controller design. The function approximations are based on the application of the universal function approximation feature of artificial neural networks.

The nonlinear AVSS can be modelled in discretized form as

$$
\begin{aligned}
y(k)= & f\left[y(k-1), \ldots, y\left(k-n_{a f}\right), u(k-2),\right. \\
& \left.\ldots, u\left(k-n_{b f}\right)\right]+g[y(k-1), \ldots, \\
& y\left(k-n_{a g}\right), u(k-2), \ldots, \\
& \left.u\left(k-n_{b g}\right)\right] u(k-1),
\end{aligned}
$$




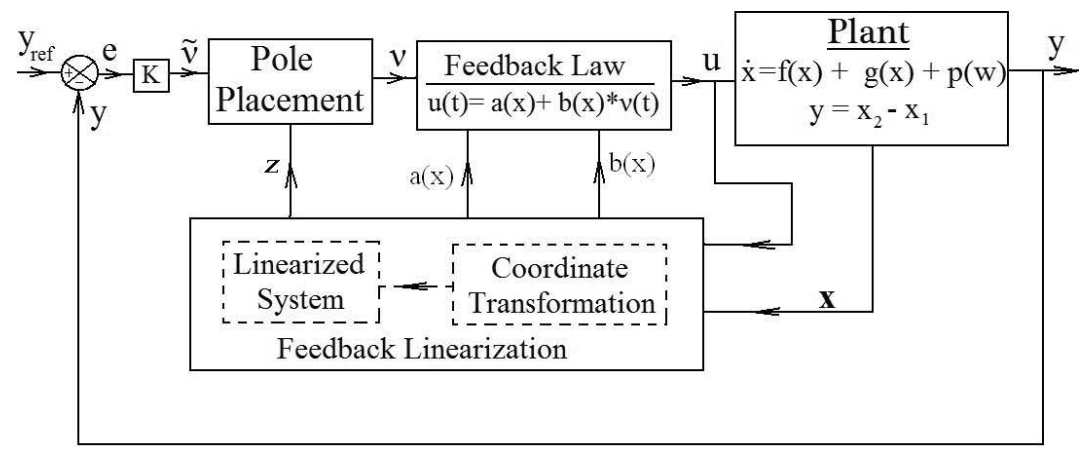

Fig. 4. Input-output feedback linearization with pole placement.

where $f[\cdot]$ and $g[\cdot]$ are two different nonlinear functions; $n_{a f}$ and $n_{b f}$ represent the number of past output and input data sample to be controlled based on the approximation of the function $f[\cdot]$ at every sampling instance; $n_{a g}$ and $n_{b g}$ represent respectively the numbers of past output and input data samples to be controlled based on the approximation of the function $g[\cdot]$ at every sampling instance.

Two different multilayer perceptron neural networks (MLPNNs) are trained off-line to approximate the two nonlinear functions. This is implemented in the NNSYSID toolbox using the function NNIOL. Equations (34) and (43) can be rearranged in the following form:

$$
\mathbf{u}(t)=\frac{\nu(t)-\tilde{f}[\mathbf{x}(t)]}{\tilde{g}[\mathbf{x}(t)]} .
$$

(44) is given in discretized form as

$$
\begin{gathered}
\mathbf{u}(k)=\frac{n(k)}{d(k)}, \\
n(k)=\nu(k)-\tilde{f}\left[y(k-1), \ldots, y\left(k-n_{a f}\right),\right. \\
\left.u(k-2), \ldots, u\left(k-n_{b f}\right)\right] \\
d(k)=\tilde{g}\left[y(k-1), \ldots, y\left(k-n_{a g}\right),\right. \\
\left.u(k-2), \ldots, u\left(k-n_{b g}\right)\right]
\end{gathered}
$$

such that (44) takes the form of (19) and

$$
\mathbf{a}(\mathbf{x})=-\frac{\tilde{f}[\mathbf{x}(t)]}{\tilde{g}[\mathbf{x}(t)]}, \quad \mathbf{b}(x)=\frac{1}{\tilde{g}[\mathbf{x}(t)]},
$$

where $\tilde{g}[\mathbf{x}(t)]=\mathcal{L}_{g} \mathcal{L}_{f}^{3} \neq 0, \tilde{f}[\mathbf{x}(t)]=\mathcal{L}_{f}^{4} \mathbf{h}(\mathbf{x}), \nu=$ $\nu(t)$ and $\mathbf{u}=\mathbf{u}(t)$

The NNIOL feedback law given in (46) is implemented within a closed-loop system that consists of the linear controller (which is a pole placement controller in this case, as shown in Fig. (4) and the plant using neural networks as presented in Fig. 5.

MLPNN structures are chosen for both networks because of their simplicity and associated computational ease. The number of neurons in the hidden layer of each

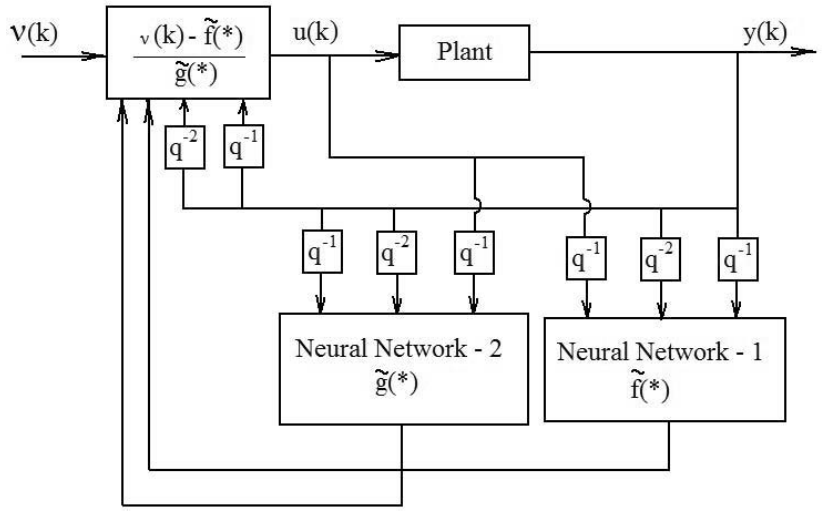

Fig. 5. Neural network based discrete input-output linearization (NNIOL) architecture.

network is computed from the summation of their respective number of past outputs and inputs, and the time delay. Thus, the hidden layer of the $\mathbf{f}$-network has five neurons while the hidden layer of the g-network has three neurons. The difference in the number of hidden layer neurons is due to the difference in the level of complexity of functions $\mathbf{f}$ and $\mathbf{g}$, as evident from (8) and (9). Figure 5 illustrates function approximation based on (46). The input layers contain two neurons and a bias in each case, and the tangent hyperbolic activation function is given by

$$
f(x)=\tanh (x)=\frac{e^{x}-e^{-x}}{e^{x}+e^{-x}} .
$$

The output layer contains one neuron with linear activation function (Norgaard et al., 2003). The training parameters for the function approximations are listed in Table 3 .

\section{Discussion and simulation results}

The NNFBL and PID controllers were applied to an AVSS nonlinear model with the actuation force generated by an electro-hydraulic actuator. A variable but preset control input in the form of voltage (which was $\leq 10 \mathrm{~V}$ ) was sup- 
plied to the servo-valve to generate the actuation force at the piston.

Table 4 presents a summary of the designed controllers' performance against specified requirements. The suspension travel responses in Figs. 6 and 7 show good command tracking by both controllers. Both controllers have no overshoots and reached a zero steady state error before the next transition point. Both controllers also have rise times that are less than the specified rise time. The rise time for the PID controller is about twice as much as the one for the NNFBL controller.

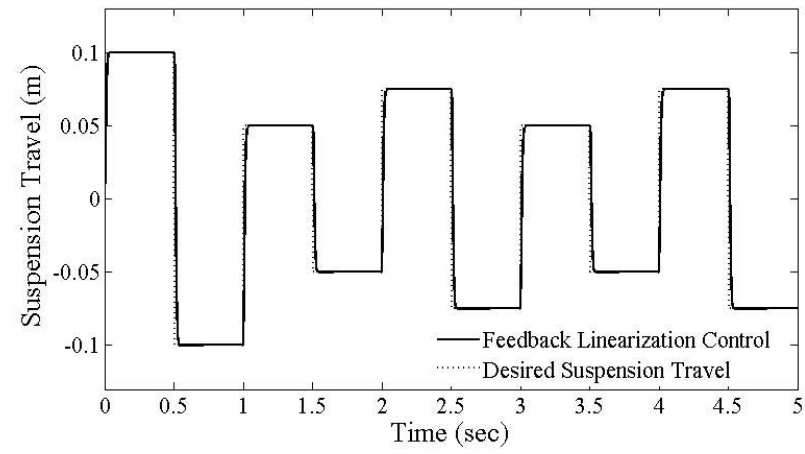

Fig. 6. Suspension travel tracking for neural network based feedback linearization control.

Figure 8 shows the control input for the NNFBL controller. It is ranged between (approximately) $-0.01 \mathrm{~V}$ and $-0.157 \mathrm{~V}$. The control input was characterized by chattering at the transition points. In Fig. 9, the control input for the PID controller varied between $1.1 \mathrm{~V}$ and $-1.45 \mathrm{~V}$ (approximately) spikes at the transition points but steady constant values which also vary between $+0.1 \mathrm{~V}$ and $-0.1 \mathrm{~V}$.

Table 3. Parameters for the neural network model

\begin{tabular}{|l|c|}
\hline Parameters & Value \\
\hline \hline Total number of samples & 5000 \\
Number of layers & 2 \\
Number of Iterations & 500 \\
Time delay & 1 \\
Training algorithm & Levenberg-Marquandt \\
Total sampling time & $5 \mathrm{~s}$ \\
Sampling time, $T_{s}$ & $0.001 \mathrm{~s}$ \\
& \\
f-network & \\
Number of hidden layer & 5 \\
neurons & 2 \\
Number of past outputs, $n_{a f}$ & 2 \\
Number past inputs, $n_{b f}$ & \\
g-network & 3 \\
Number of hidden layer & 1 \\
neurons & 1 \\
Number of past outputs, $n_{a g}$ & \\
Number past inputs, $n_{b g}$ & \\
\hline
\end{tabular}

Table 4. Controller performance evaluation.

\begin{tabular}{|l|c|c|c|}
\hline $\begin{array}{l}\text { Performance } \\
\text { parameters }\end{array}$ & $\begin{array}{c}\text { Specified } \\
\text { value }\end{array}$ & FBL & PID \\
\hline \hline Over-shoot & $\leq 5 \%$ & $0 \%$ & $0 \%$ \\
Rise time [s] & $\leq 0.1$ & 0.015 & 0.033 \\
Steady state error & $0 \%$ & $0 \%$ & $0 \%$ \\
Control input [V] & \pm 10 & $0.017 \%$ & $0.149 \%$ \\
Performance index $J$ & min & 0.020 & 0.432 \\
\hline
\end{tabular}

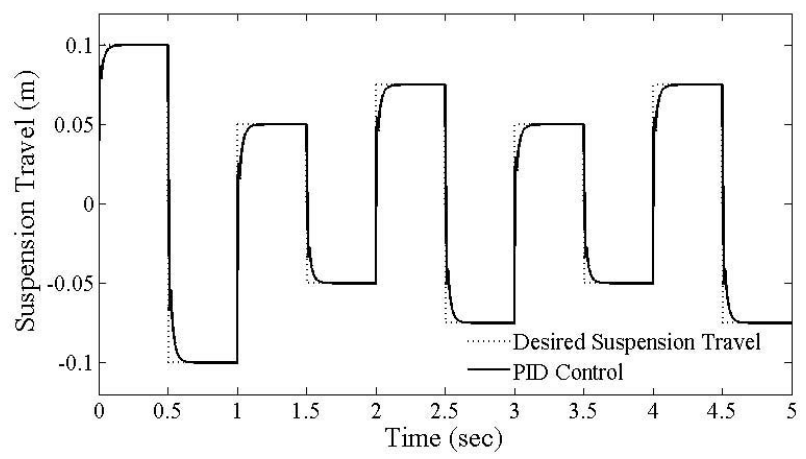

Fig. 7. Suspension travel tracking for PID control.

The NNFBL control was achieved at a much lower cost than the PID control. Table 4 shows that the NNFBL controller exhibits better minimization of the performance index.

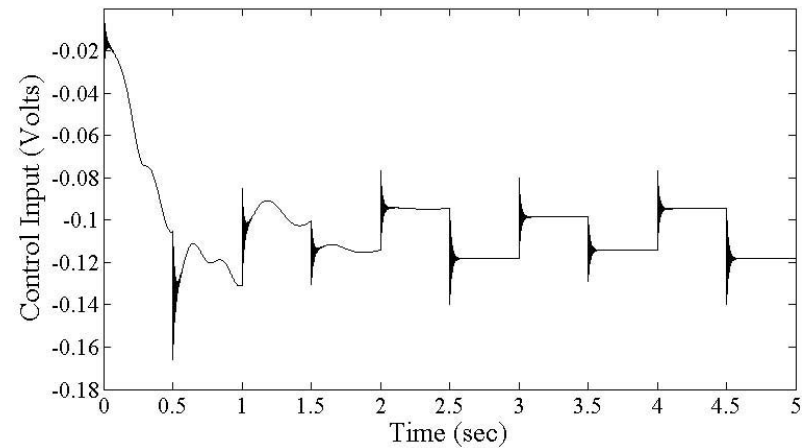

Fig. 8. Feedback linearization control input.

\section{Conclusion}

PID and NNFBL controllers were designed for a nonlinear active suspension system. The NNFBL controller shows better tracking of the desired output in the presence of a deterministic disturbance input. There were no overshoots at the transition points of the desired output being tracked. The controllers were characterized by the following:

1. lower rise times than the specified value,

2. chattering in the supply voltage signal for the NNFBL controller, 


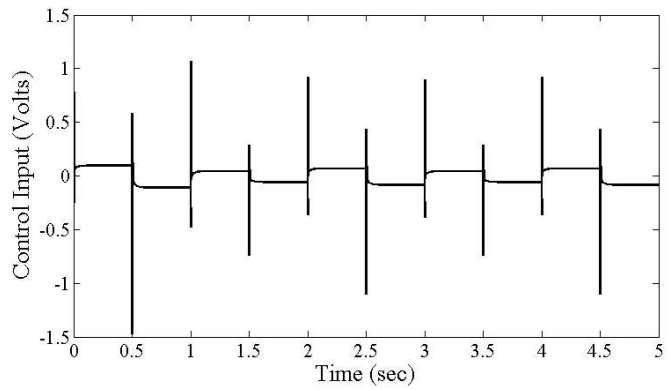

Fig. 9. PID control input.

3. lower supply voltage requirement by NNFBL than by the PID controller throughout the tracking process, and

4. the supply voltage to the PID controller characterized by intermittent spikes, respectively.

Finally, the superior performance of the NNFBL controller in adapting to deterministic road disturbance as against generic PID was presented. The NNFBL controller combines multilayer perceptron neural networks with the direct adaptive-feedback linearization in cancelling system nonlinearities and enabling the application of a linear control law.

\section{References}

Al-Holou, N., Lahdhiri, T., Joo, D.S., Weaver, J. and Al-Abbas, F. (2002). Sliding mode neural network inference fuzzy logic control for active suspension system, IEEE Transactions on Fuzzy Systems 10(2): 234-245.

Biglarbegian, M., Melek, W. and Golnaraghi, F. (2008). A novel neuro-fuzzy controller to enhance the performance of vehicle semi-active suspension systems, Vehicle System Dynamics 46(8): 691-711.

Boukezzoula, R., Galichet, S. and Foulloy, L. (2007). Fuzzy feedback linearizing controller and its equivalence with fuzzy nonlinear internal model control structure, International Journal of Applied Mathematics and Computer Science 17(2): 233-248, DOI: 10.2478/v10006-007-0021-4.

Boutalis, Y.S. (2004). Neural network approaches for feedback linearization, Journal of Control Engineering and Applied Informatics 6(1): 15-26.

Buckner, G.D., Schuetze, K.T. and Beno, J.H. (2001). Intelligent feedback linearization for active vehicle suspension control, Journal of Dynamic Systems, Measurement and Control: Transactions of ASME 123(4): 727-733.

Cao, J., Liu, H., Li, P. and Brown, D. (2008). State of the art in vehicle active suspension adaptive control systems based on intelligent methodologies, IEEE Transactions of Intelligent Transportation Systems 9(3): 392-405.
Chantranuwathana, S. and Peng, H. (2004). Adaptive robust force control for vehicle active suspension, International Journal of Adaptive Control and Signal Processing 18(2): 83-102.

Christophe, L., Swevers, J. and Sas, P. (2005). Robust linear control of an active suspension on a quarter car test-rig, Control Engineering Practice 13(5): 577-586.

Dahunsi, O.A., Pedro, J.O. and Nyandoro, O.T. (2009). Neural network-based model predictive control of a servohydraulic vehicle suspension system, Proceedings of 2009 IEEE AFRICON, Nairobi, Kenya, pp. 1-6, DOI: 10.1109/AFRCON.2009.5308111.

Dahunsi, O.A., Pedro, J.O. and Nyandoro, O.T. (2010a). Neural network-based PID control of a servo-hydraulic vehicle suspension system, Proceedings of the 7th South African Conference on Computational and Applied Mechanics (SACAM10), Pretoria, South Africa, pp. 1-6.

Dahunsi, O.A., Pedro, J.O. and Nyandoro, O.T. (2010b). System identification and neural network based PID control of servo-hydraulic vehicle suspension system, SAIEE Africa Research Journal 101(3): 93-105.

D'Amato, F.J. and Viassolo, D.E. (2000). Fuzzy control for active suspensions, Mechatronics 10(1): 897-920.

Deng, J., Becerra, V.M. and Stobart, R. (2009). Input constraints handling in an MPC/feedback linearization scheme, International Journal of Applied Mathematics and Computer Science 19(2): 219-232, DOI: 10.2478/v10006-009-00182.

Du, H. and Zhang, N. (2008). Multiobjective static output feedback control design for vehicle suspensions, Journal of System Design and Dynamics 2(1): 228-239.

Du, H. and Zhang, N. (2009). Static output feedback control for electrohydraulic active suspensions via T-S fuzzy model approach, Journal of Dynamic Systems, Measurement and Control: Transactions of ASME 131(5): 0510041-051004-11.

Ehtiwesh, I.A.S. and Dorovic, Z. (2009). Comparative analysis of different control strategies for electro-hydraulic servo systems, Proceedings of the World Academy of Science, Engineering and Technology 56(6): 906-909.

Eski, I. and Yildrim, S. (2009). Vibration control of vehicle active suspension system using a new robust neural network control system, Simulation Modelling Practice and Theory 17(5): 778-793.

Fallah, M.S., Bhat, R. and Xie, W. (2009). $H_{\infty}$ robust control of active suspensions: A practical point of view, Proceedings of the 2009 American Control Conference, St Louis, MO, USA, pp. 1385-1390.

Feng, J.Z., Li, J. and Yu, F. (2003). GA-based PID and fuzzy logic control for active vehicle suspension system, International Journal of Automotive Technology 4(4): 181-191.

Fiahlo, I. and Balas, G.J. (2002). Road adaptive active suspension using linear parameter-varying gain-scheduling, IEEE Transactions on Control Systems Technology 10(1): 43-54. 
Gao, B., Tilley, D.G., Williams, R.A., Bean, A. and Donahue, J. (2006). Control of hydropneumatic active suspension based on a non-linear quarter-car model, Proceedings of the Institute of Mechanical Engineers, Part I: Journal of Systems and Control Engineering 220(1): 75-31.

Gao, Z. (2002). From linear to nonlinear control means: A practical progression, ISA Transactions 41(2): 177-189.

Garces, F.R., Becerra, V.M., Kambhampti, C. and Warwick, K. (2003). Strategies for Feedback Linearisation: A Dynamic Neural Network Approach, Springer, London.

Gaspar, P., Szaszi, I. and Bokor, J. (2003). Active suspension design using linear parameter varying control, International Journal of Autonomous Systems 1(2): 206-221.

Goodwin, G.C., Rojas, O. and Takata, H. (2001). Nonlinear control via generalized feedback linearization using neural networks, Asian Journal of Control 3(2): 79-88.

Hada, M.K., Menon A. and Bhave S.Y. (2007). Optimisation of an active suspension force controller using genetic algorithm for random input, Defence Science Journal 57(5): 691-706.

Hagan, M.T. and Demuth, H.B. (1999). Neural networks for control, American Control Conference, San Diego, CA, USA, pp. $1642-1656$.

Hassanzadeh, I., Alizadeh, G., Shiirjoposht, N. P. and Hashemzadeh, F. (2010). A new optimal nonlinear approach to half car active suspension control, IACSIT International Journal of Engineering and Technology 2(1): $78-84$.

He, Y. and McPhee, J. (2005). A design methodology for mechatronic vehicles: Application of multidisciplinary optimization, multibody dynamics and genetic algorithms, Vehicle Systems Dynamics 43(10): 697-733.

Hrovat, D. (1997). Survey of advanced suspension developments and related optimal control applications, Automatica 33(10): 1781-1817.

Isidori, A. (1989). Nonlinear Control System, Springer-Verlag, Berlin.

Jelali, M. and Kroll, A. (2003). Hydraulic Servo-Systems: Modelling, Identification and Control, Springer-Verlag, London.

Jin, Y. and Yu, D.J. (2008). Adaptive neuron control using an integrated error approach with application to active suspensions, International Journal of Automotive Technology 9(3): 329-335.

Kar, I. and Behera, L. (2009). Direct adaptive neural control for affine nonlinear systems, Applied Soft Computing 9(2): 756-764.

Koshkouei, A.J. and Burnham, K.J. (2008). Sliding mode controllers for active suspensions, Proceedings of the 17th IFAC World Congress, COEX, Seoul, Korea, pp. 1-6.

Kumar, M.S. (2008). Development of active suspension system for automobiles using PID controller, Proceedings of the World Congress on Engineering, WCE 2008, London, UK, pp. 1472-1477.
Kumar, M.S. and Vijayarangan, S. (2007). Analytical and experimental studies on active suspension system of light passenger vehicle to improve ride comfort, Mechanika 65(3): 34-41.

Kuo, Y. and Li, T.S. (1999). GA-based fuzzy PI/PD controller for automotive active suspension system, IEEE Transactions on Industrial Electronics 46(6): 88-100.

Lufty, O.F., Noor, S.B.M., Marhaban, M.H. and Abbas, K.A. (2009). A geneticatlly trained adaptive neuro-fuzzy inference system network utilized as a PID-like feedback controller for non-linear systems, Proceedings of the Institute of Mechanical Engineers, Part I: Journal of Systems and Control Engineering 223(3): 289-429.

Nakkarat, P. and Kuntanapreeda, S. (2009). Observer-based backstepping force control of an electrohydraulic actuator Control Engineering Practice 17(8): 895-902.

Norgaard, M., Ravn, O., Poulsen, N.K. and Hansen, L.K. (2003). Neural Networks for Modelling and Control of Dynamic Systems: A Practitioner's Handbook, Springer, Boston, MA.

O'Dwyer, A. (2006). Handbook of PI and PID Controller Tuning Rules, Imperial College Press, London.

Pedro, J.O. (2003). Design and performance of an active vehicle suspension system, Proceedings of the 2nd International Conference on Applied Mechanics and Materials, ICAMM 2003, Durban, South Africa, pp. 203-209.

Pedro, J.O. (2007). $H_{2}$-LQG/LTR controller design for active suspension systems, $R$ and D Journal of the South African Institution of Mechanical Engineering 23(2): 32-41.

Pedro, J.O. and Mgwenya, T.R. (2004). LQR control of a full car active suspension with actuator dynamics, Proceedings of the 4th South African Conference on Applied Mechanics, SACAM'04, Johannesburg, South Africa, pp. 1-9.

Poursamad, A. (2009). Adaptive feedback linearization control of antilock braking system using neural networks, Mechatronics 19(5): 767-773.

Poussot-Vassal, C., Sename, O., Dugard, L., Gaspar, P., Szabo, Z. and Bokor, J. (2006). Multi-objective qLPV $H_{\infty} / H_{2}$ control of a half vehicle, Proceedings of the 10th MINI Conference on Vehicle System Dynamics, Identification and Anomalies, Budapest, Hungary, pp. 1-6.

Ryu, S., Kim, Y. and Park, Y. (2008). Robust $H_{\infty}$ preview control of an active suspension system with norm-bounded uncertainties, International Journal of Automotive Technology 9(5): 585-592.

Salem, A.A.A. and Aly, A.A. (2009). Fuzzy control of a quarter-car suspension systems, Proceedings of the World Academy of Science, Engineering and Technology (53): 258-263.

Seo, J., Venugopal, R. and Kenne, J. (2007). Feedback linearization based control of a rotational hydraulic drive, Control Engineering Practice 15(12): 1495-1507.

Sharkawy, A.B. (2005). Fuzzy and adaptive fuzzy control for the automobiles' active suspension system, Vehicle Systems Dynamics 43(10): 795-806. 
Shen, X. and Peng, H. (2003). Analysis of active suspension systems with hydraulic actuators, Proceedings of the 2003 IAVSD Conference, Atsuigi, Japan, pp. 1-10.

Shi, J., Li, X.W. and Zhang, J.W. (2010). Feedback linearization and sliding mode control for active hydropneumatic suspension of a special-purpose vehicle, Proceedings of the Institute of Mechanical Engineers, Part D: Journal of Automobile Engineering 211(3): 171-181.

Shirahatt, A., Prasad, P.S.S., Panzade, P. and Kulkarni, M.M. (2008). Optimal design of passenger car suspension for ride and road holding, Journal of the Brazillian Society of Mechanical Science and Engineering 30(1): 66-76.

He, S., Reif, K. and Unbehauen, R. (1998). A neural approach for control of nonlinear systems with feedback linearization, Control Engineering Practice 9(6): 1409-1421.

Slotine, J.J. and Li, W. (1991). Applied Nonlinear Control, Prentice Hall, Englewood Cliffs, NJ.

Yagiz, N., Sakman, L.E. and Guclu, R. (2008). Different control applications on a vehicle using fuzzy logic control, Sadhana 33(1): 15-25.

Yesildirek, A. and Lewis, F.L. (1995). Feedback linearization using neural networks, Automatica 31(11): 1659-1664.

Yoshimura, T. and Teramura, I. (2005). Active suspension control of a one-wheel car model using single input rule modules fuzzy reasoning and a disturbance observer, Journal of Zhejiang University: Science 6A(4): 251-256.

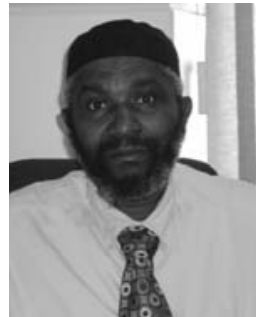

Jimoh Olanrewaju Pedro received the M.Sc and $\mathrm{Ph} . \mathrm{D}$. degrees in aeronautical engineering from the Warsaw University of Technology, Poland, in 1986 and 1992, respectively. He was a post-doctoral research fellow at the Institute of Aviation in Warsaw (1993-1994). He is currently a senior lecturer with the School of Mechanical, Industrial and Aeronautical Engineering, University of the Witwatersrand, Johannesburg, South Africa. He is the author of more than 30 papers in refereed journals and conference proceedings. His research interests include applications of optimal control, robust control, nonlinear control and computational intelligence to aerospace vehicles and mechatronic systems.

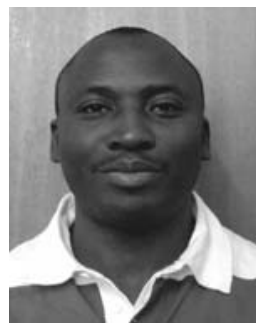

Olurotimi Akintunde Dahunsi received the B.Eng. and M.Eng. degrees in mechanical engineering in 1994 and 2001, respectively. He is currently pursuing his $\mathrm{Ph} . \mathrm{D}$. in mechanical engineering at the University of the Witwatersrand. His research interest includes intelligent control techniques, vehicle dynamics and vibration analysis.

Received: 30 March 2010 Revised: 26 September 2010 